\title{
Orientation dependent surface stabilization on flame deposited diamond single crystals
}

\author{
J.J. Schermer ${ }^{*}$, W.J.P. van Enckevort, L.J. Giling \\ Research Institute for Materials, University of Nijmegen, Toernooiveld, 6525 ED Nijmegen, The Netherlands
}

Received 30 September 1994; manuscript received in final form 18 November 1994

\begin{abstract}
In this study a model for the orientation dependent surface stabilization of diamond grown from the vapour phase is presented. The model is based on a first order broken bond analysis of the diamond crystal with additional considerations about surface reconstruction by dimer formation. Due to the formation of dimers, surface stabilization is expected for orientations corresponding to Miller indices $\{h h k\}_{h<k}$ in the $\langle 110\rangle$ zones. It is demonstrated that this model gives a good description of growth phenomena like facets, curved bands and microfaceting, observed on homoepitaxially grown diamond samples. These specimens are obtained by flame deposition on cylindrical type IIa natural diamond substrates with $\{111\},\{110\}$ or $\{001\}$ top faces. In conformation with the model the surfaces $\{h h k\}_{h<k}$ between $\{111\}$ and $\{113\}$ appear to be stabilized in one direction, while a discrete number of orientations between $\{113\}$ and $\{001\}$ are subject to two-dimensional stabilization. All other surfaces are stabilized by the development of microfacets which also have orientations close to $\{h h k\}_{h \leq k}$, as was determined by laser reflectometry. In addition it is shown that differences in the orientation dependent incorporation of nitrogen as nitrogen-vacancy pairs in the diamond lattice is consistent with the described model. For this purpose a single crystalline diamond layer grown on top of a hemispherical diamond substrate by the flame technique was investigated by cathodoluminescence topography.
\end{abstract}

\section{Introduction}

Recently homoepitaxial diamond growth by the acetylene-oxygen combustion flame technique [1] was reported on cylindrically shaped type IIa natural diamond substrates with $\{001\},\{110\}$ and $\{111\}$ top faces [2-7]. Infrared, Raman and luminescence spectroscopy demonstrated that the quality of the $\{001\}$ and $\{111\}$ samples is comparable to that of type IIa natural diamond, although

* Corresponding author. crystallographic imperfections like cracks and twins were observed for the $\{111\}$ samples $[5,6]$. The quality of the $\{110\}$ specimens is slightly less, amongst others due to an enhanced incorporation of hydrogen and nitrogen [5]. The samples exhibit facets and curved bands in the $\{h h k\}_{h \leq k}$ parts of the $\langle 110\rangle$ zones. Orientations between $\{111\}$ and $\{113\}$ in this zone appeared to be stabilized in one direction, while the stabilization for orientations between $\{113\}$ and $\{001\}$ has a more two-dimensional character. All other orientations, including the $\{h h k\}_{h>k}$ parts of the $\langle 110\rangle$ zones, like $\{110\}$, have rough, pitted surfaces due to the develop- 
ment of microfacets. The observed stabilization of surfaces in the $\{h h k\}_{h<k}$ parts of the $\langle 110\rangle$ zones instead of those in the $\{h h k\}_{h>k}$ parts, is not expected from a periodic bond chain analysis of the diamond crystal [8].

Gardeniers et al. used a first order broken bond analysis to describe the orientation dependent homoepitaxial deposition of silicon on hemispheres $[9,10]$. In order to explain the observed growth phenomena for orientations with Miller indices $\{h h k\}_{h<k}$, they put forward additional considerations about surface reconstruction and adsorption of species. Although this model was derived for silicon chemical vapour deposition (CVD) under near equilibrium conditions, it is applicable to describe the surface stabilization of vapour phase grown crystals with a diamond structure in general. Even for diamond growth, which is considered to be a process far from equilibrium, the model proved to be. successful in the qualitative description of the temperature dependent occurrence of $\{113\}$ facets on homoepitaxially grown single crystals [4]. In the present study a model will be derived according

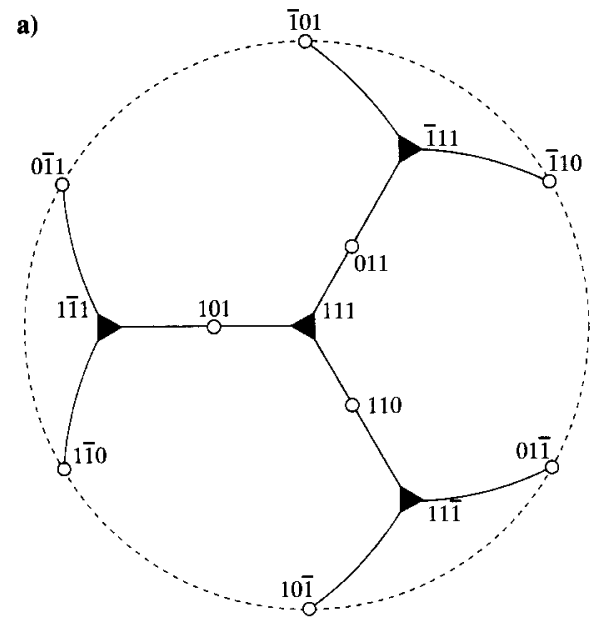

to the same principles which gives a more detailed description of the possible two-dimensional stabilization for a discrete number of surfaces with orientations between $\{113\}$ and $\{001\}$ in the $\langle 110\rangle$ zones. This model will be used as a starting point to discuss the observed features like facets, curved bands and microfacets on the diamond single crystals mentioned above. In addition it will be demonstrated that there is a relation between the surface stabilization as described by the model and the orientation dependent incorporation of nitrogen as nitrogen-vacancy pairs into the diamond lattice.

\section{Theory of surface stabilization}

In general, $\{h k l\}$ facets on a crystal will develop for those orientations that have a connected network of atoms or molecules within the repeat distance $d_{h k l}$ [12]. Following the GibbsWulff theorem, the development of these facets is possible because they have a lower growth rate than all their neighbouring orientations. On such

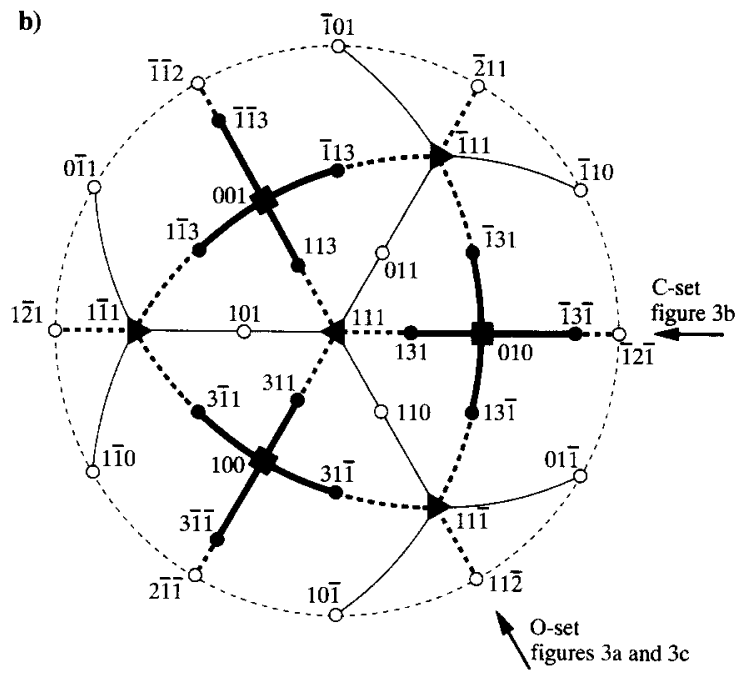

Fig. 1. Stereographic projection on the (111) plane showing zones with orientations (lines) and faces (grey markers) on a diamond type crystal which are expected to be stabilized in one and two directions, respectively. (a) According to the PBC theory. (b) According to the PBC theory with additional considerations about dimer formation between the surface atoms. The thin and bold (see text for the difference between the full and broken bold lines) lines indicate the $\{h h k\}_{h>k}$ and $\{h h k\}_{h<k}$ parts of the $\langle 110\rangle$ zones, respectively. The faces indicated by the open markers are subject to discussion but are not stabilized in two directions. The periphery of the projection is outlined by the thin broken line. 
(flat) F-faces growth proceeds layer by layer due to a positive step free energy. Orientations which contain periodic bond chains (PBCs) of connected atoms or molecules in only one direction within their surface slice $d_{h k l}$, are (stepped) Sfaces. For a set of these orientations in one zone, a band which is flat along the PBCs but curved in the other directions will develop. Other orientations for which the surfaces are kinked (K-faces) have no tendency to flatten and appear as rough surfaces.

The only PBCs for the diamond structure are the zigzag chains along $\langle 110\rangle$. Orientations in zones parallel to these PBCs are potential S-faces, while the $\{111\}$ and $\{001\}$ faces where these zones intersect are potential F-faces [13]. However, the $\{001\}$ surface is kinked because it does not contain any PBC within its repeat distance $d_{004}$. Consequently, the faces in the $\langle 110\rangle$ zones between $\{111\}$ and $\{001\}$, which can be described by Miller indices $\{h h k\}_{h<k}$, are not S-faces because they are partly kinked [8]. Therefore only the faces in the $\langle 110\rangle$ zones between $\{111\}$ and $\{110\}$, which can be denoted by $\{h h k\}_{h>k}$, are stepped, while $\{111\}$ are the only F-faces of the diamond crystal. Neglecting entropy effects, the surface free energy can be assumed to be proportional with the density of dangling bonds at a crystal surface [14-16]. Furthermore, considering the crystal surface merely as a simple truncation of the bulk crystal, a first order approximation for the surface free energy as a function of the crystallographic orientation can be derived. In Fig. 1a a schematic representation of this three-dimensional Herring [17] plot for crystals with a diamond lattice is given in a stereographic projection [18]. This approach appears consistent with the PBC theory since the plot shows grooves along the $\{h h k\}_{h>k}$ parts of the $\langle 110\rangle$ zones (full lines) connecting the eight absolute minima at geometrical positions corresponding to $\{111\}$ facets (triangles).

In contrast to this description, besides the $\{111\}$ facets also flat $\{001\}$ facets are observed on diamond layers obtained by chemical vapour phase deposition [2-6,19-21]. The presence of these facets was explained by a very persistent $(2 \times 1)$ surface reconstruction due to the formation of dimer bonds, which gives a significant decrease in dangling bond density and creates a positive step free energy [22-24]. Accounting for this surface reconstruction, six additional minima with orientations corresponding to the $\{001\}$ faces (squares in Fig. 1b), appear in the polar plot of the dangling bond density (surface free energy).

In principle similar dimers can also be formed on the surfaces in the $\langle 110\rangle$ zones between $\{001\}$ and $\{111\}$. Therefore each of the cubic faces is connected with four adjacent $\{111\}$ minima by grooves along the $\{h h k\}_{h<k}$ orientations (full and dashed thick lines in Fig. 1b). The surface reconstruction of these orientations will be discussed below. This discussion is restricted to the orientations between (111) and (001) since all the other $\{h h k\}_{h \leq k}$ grooves are symmetrically related to this zone. After projection of the crystal structure along the $[1 \overline{1} 0]$ PBC, the orientations are defined by their angle $\theta$ with respect to the (111) face. The surfaces in the zone are subject to reconstruction by dimer formation parallel $\left(R_{\|}\right)$or perpendicular $\left(R_{\perp}\right)$ to the [110] PBCs. For each surface atom with two dangling bonds one will remain after $R_{\|}$(see Fig. 2b). As shown in Fig. 2a this results in a continuous decrease of the dangling bond density $\left(C_{\mathrm{db}}\right)$ from (111) towards (001) compared to the unreconstructed case [10]. For almost all orientations an energetically more favourable situation is obtained by $R_{\perp}$ which therefore will be elaborated in more detail.

The $(h h k)_{h \leq k}$ zone can be divided in two parts as is also indicated by the dashed and full thick lines in Fig. 1b. Surfaces with orientations between (111) and (113) can be considered to be built up from (111) terraces with steps formed by two rows of atoms having an (001) orientation (see Fig. 2b). Only the surface atoms in these steps can form dimers perpendicular to the [110] PBCs and after reconstruction only one of the three dangling bonds in the step is left. Therefore $C_{\mathrm{db}}$ continuously decreases with increasing step density from $100 \%$ of its value before reconstruction for the (111) up to $33 \%$ for the (113) where the width of the (111) terraces is reduced to a single row of atoms without a dangling bond. Beyond the (113) orientation up to (001), the surfaces can be considered to be built up from 

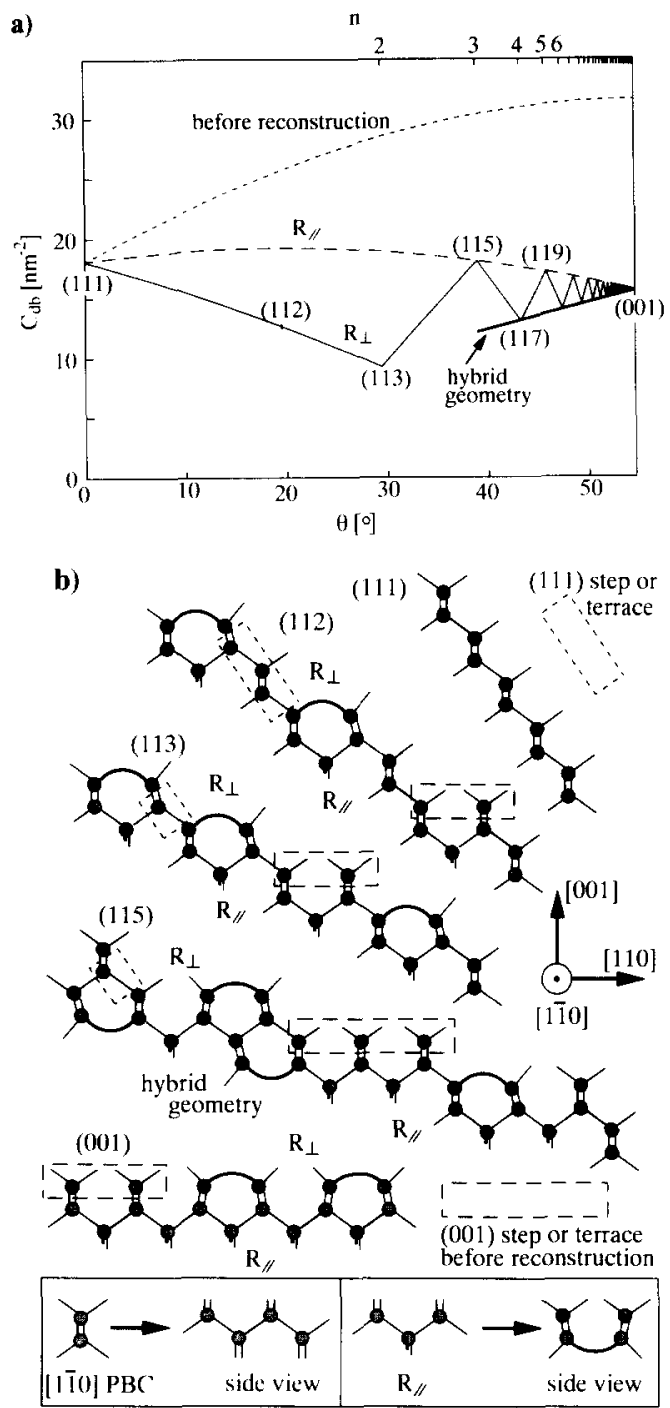

Fig. 2. Reconstruction of the surfaces $\{h h k\}_{h<k}$ by dimer formation. (a) The dangling bond densities $\left(C_{\mathrm{db}}\right)$ before and after surface reconstruction according to several dimer geometries (see text) for surfaces in the [110] zone as a function of their angle $\theta$ with the (111) face. (b) The surface structures for several orientations in this zone projected along [110].

single atom (111) steps without a dangling bond and (001) terraces. These terraces are formed by a discrete number of $n(n \geq 2)$ parallel [11̄0] PBCs (see Fig. 2b). The atoms in the first PBC (adjacent to the (111) step) of the terrace have one and those in each additional PBC have two dangling bonds, see the dashed boxes for the
(113) and (115) orientations in Fig. 2b. After reconstruction $C_{\mathrm{db}}$ is reduced to a fraction of $(n-1) /(2 n-1)$ of its original value for even values of $n$ and to $n /(2 n-1)$ for odd values, corresponding to (local) minima e.g. (113) and maxima e.g. (115), respectively, as shown in Fig. 2a. In the idealized situation of equally spaced steps, a surface with $n$ PBCs in each (001) terrace corresponds to an (1 $12 n-1)$ face. Surfaces with intermediate orientations can be considered as being built up from a linear combination of the two adjacent discrete terrace widths and according to this they have a $C_{\mathrm{db}}$ which is a proportional average.

For orientations between (115) and (001) besides $R_{\|}$and $R_{\perp}$, a hybrid configuration is possible with dimers parallel to the [110] PBCs at the terraces but one row of dimers perpendicular to these PBCs in the step (see Fig. 2b). This configuration with double height steps, which according to the notation of Chadi [25] are of type $\mathrm{D}_{\mathrm{B}}$ with "rebonded edge geometry", reduces $C_{\mathrm{db}}$ to $(n-$ 1) $/(2 n-1)$ times its value before reconstruction for all $n \geq 3$ (see Fig. 2a). In accordance with the lower values of $C_{\mathrm{db}}$, for silicon $\{001\}$ vicinal faces these $D_{B}$ steps were calculated to be energetically preferable above $D_{A}$ steps [25], which occur in the case of $R_{\perp}$ with only dimer formation perpendicular to the [11̄0] PBCs. Although energetically favourable, the hybrid geometry only partly contributes to stabilization perpendicular to the [110] PBCs. Furthermore according to this geometry $C_{\mathrm{db}}$ as a function of $\theta$ has no (local) minima besides (115), therefore no two-dimensional stabilization is expected for orientations between (115) and (001).

Although the effect of reconstruction on the surface free energy is partly counter balanced by increased bond strains [10,15], it can be concluded that the minimum energy for $\{h h k\}_{h<k}$ faces is obtained by $R_{\perp}$ for orientations between $\{111\}$ and $\{113\}$ and by the hybrid configuration for the orientations between $\{115\}$ and $\{001\}$. Orientations between $\{113\}$ and $\{115\}$ can be considered to be stabilized by a linear combination of both. This, in essence is, a zero Kelvin model. In practice, however, the situation is determined by non-equilibrium statistical mechanisms: non- 
equally sized terraces will be formed and all possible dimer geometries are expected to coexist with some non-reconstructed regions. Therefore $\{111\},\{001\}$ and the orientations corresponding with the most pronounced minima according to $R_{\perp}$ and the $\{115\}$ according to the hybrid geometry (see Fig. 2a), might be subject to stabilization in two directions and thus appear as planar facets. Recently, scanning tunnelling microscopy (STM) indeed revealed the occurrence of $D_{B}$ steps [26], $D_{A}$ steps [23] as well as non-reconstructed areas [23] on exact and misoriented $\{001\}$ diamond layers.

In addition to dimer formation, a reduction of dangling bonds at the crystal surface can be obtained by the adsorption of species from the gas phase [27] at the dangling bonds which remain after surface reconstruction. Therefore the minima shown in Fig. 2a become less pronounced upon increasing adsorption and the morphological importance of the orientations corresponding with these minima will decrease. Due to this process of "chemical roughening" the surfaces tend to become stepped and curved perpendicular to the $\langle 110\rangle$ PBCs [10]. Diamond CVD is a process far from equilibrium with high concentrations of atomic hydrogen and hydrocarbon radicals in the gas phase. The saturation of the remaining dangling bonds on the reconstructed diamond surface by these species is close to unity at deposition temperatures up to $1000^{\circ} \mathrm{C}[28,29]$. However, the surface coverage decreases upon lowering the applied supersaturation of hydrocarbon species in the gas phase and upon increasing deposition temperatures so that desorption of the species from the surface becomes important [10]. Under these conditions the orientations corre- sponding with the most pronounced minima in Fig. 2a might appear as flat facets as was confirmed by the occurrence of $\{113\}$ facets on flame deposited diamond single crystals at temperatures around $1200^{\circ} \mathrm{C}[4,7]$.

\section{Crystal growth and characterization}

The flame deposition set-up used to grow the samples has been described in previous work $[6,30]$. An accurate regulation of the deposition temperature is obtained by soldering of the substrates onto a water cooled substrate holder with a high temperature soldering material. The flows of the high purity source gases oxygen and acetylene are regulated by mass flow controllers. The flame was generated by a welding torch using burner tips with an opening of 1.4 or $1.6 \mathrm{~mm}$ in diameter. The samples were grown using both laminar and turbulent flames. Since for both flames the differences in diamond growth were marginal compared to differences obtained by changes in the total gas flow and the deposition temperature [6], no distinction between them will be made.

Crystal growth in general is mainly determined by the deposition temperature $\left(T_{\mathrm{d}}\right)$ and the supersaturation $(\sigma)$ of the growth species at the crystal surface. In the present set-up $T_{d}$, as measured with a two-colour infrared pyrometer, can be regulated within $\pm 20^{\circ} \mathrm{C}$ absolute and $\pm 10^{\circ} \mathrm{C}$ relative [30]. A direct control of $\sigma$ at the crystal surface, however, is not possible since up to now it is not clear which species are responsible for the growth of diamond [31-35]. It is, however, obvious that it is influenced by the gas phase

Table 1

Ranges of growth conditions and results (see text for symbols)

\begin{tabular}{lllllllll}
\hline $\begin{array}{l}\text { Samples } \\
\text { (top faces) }\end{array}$ & Substrate shapes & $\begin{array}{l}\text { Dimensions } \\
(\mathrm{mm})\end{array}$ & $\begin{array}{l}T_{\mathrm{d} \pm 20} \\
\left({ }^{\circ} \mathrm{C}\right)\end{array}$ & $\begin{array}{l}S_{\mathrm{ac}} \pm 0.5 \\
(\%)\end{array}$ & $\begin{array}{l}f_{\mathrm{ox}} \pm 0.05 \\
(\mathrm{slm})\end{array}$ & $\begin{array}{l}d \pm 0.1 \\
(\mathrm{~mm})\end{array}$ & $\begin{array}{l}h_{\mathrm{d}} \pm 5 \\
(\mu \mathrm{m})\end{array}$ & $\begin{array}{l}v_{\mathrm{d}} \pm 3 \\
(\mu \mathrm{m} / \mathrm{h})\end{array}$ \\
\hline$\{111\}$ & cylindric & $\varnothing 2 \times 0.25$ & $1150-1250$ & 3.0 & 3.0 & 1.0 & $43-58$ & $14-21$ \\
$\{001\}$ & cylindric & $\varnothing 2-4 \times 0.25$ & $1150-1200$ & $3.9-5.4$ & $3.0-3.4$ & $0.5-1.5$ & $64-205$ & $34-83$ \\
$\{110\}$ & cylindric & $\varnothing 3 \times 0.3$ & 1200 & $3.9-4.6$ & 3.4 & $1.0-1.5$ & $84-266$ & $76-133$ \\
$\{110\}$ & hemispheric & $\varnothing 3.5$ & 1155 & 3.9 & 3.4 & 1.5 & $16^{\mathrm{a}}$ & 48
\end{tabular}

${ }^{a}$ Average thickness of the layer as measured by weight. 
composition and the position of the sample in the acetylene feather as defined by the distance $(d)$ between the substrate and the inner cone of the flame. The gas phase composition is determined by the absolute oxygen flow $\left(f_{o x}\right)$, the acetylene supersaturation $\left(S_{\mathrm{ac}}\right)[6,30]$ and $T_{\mathrm{d}}$. Of these parameters $S_{\mathrm{ac}}$ is expected to have the most direct relation with $\sigma$. Therefore the observed growth phenomena will be discussed in relation to $T_{\mathrm{d}}$ and $S_{\mathrm{ac}}$. A description of the substrates, the ranges of the applied growth conditions and the thicknesses $\left(h_{\mathrm{d}}\right)$ and deposition rates $\left(v_{\mathrm{d}}\right)$ of the grown layers on the top face are listed in Table 1. The $\{111\}$ samples were grown using a relatively low value of $3 \%$ for $S_{\mathrm{ac}}$, since the occurrence of defects like stacking faults, graphite-like inclusions and micro-twinning for crystal volumes grown via $\{111\}$ increases with the supersaturation $[36,37]$.

After growth the specimen were investigated by optical differential interference contrast microscopy (DICM) and scanning electron microscopy (SEM). The orientations of the facets developed along the sides of the $\{111\}$ samples were determined by measurement of their angles $\theta$ relative to the top face of the sample using a two-circle optical-reflection goniometer. The average orientations of small planar features like microfacets and steps were calculated from the angular distributions of the back reflections from a parallel laser beam incident perpendicular on the crystal surface. For this laser reflectometry (LR) technique the $632.8 \mathrm{~nm}$ line of a $\mathrm{He}-\mathrm{Ne}$ laser was used. The orientation dependent distribution of luminescent point defects in the samples was examined by the use of a commercially available 8200 MK II system for cathodoluminescence (CL) topography [38].

\section{Growth results and discussion}

Growth phenomena observed on the top faces of the cylindrical substrates have been described in detail in previous work $[5,6]$. Besides deposition of diamond on the top faces, also a number of facets and other features were seen along the sides of the substrates. Due to a lower layer thickness the $\{111\}$ samples reveal a large number of growth features which are no longer present on the $\{110\}$ and $\{001\}$ samples, because these are in a further stage of development.

\subsection{Growth on $\{111\}$ substrates}

For the $\{111\}$ samples a curved band with macro steps and more or less flat features was developed along the rim of the top face. This circular band is interrupted by six sets of broad, connected facets more or less vertical along the sides of the sample. Fig. 3 shows the curved band and the sets of facets on a $\{111\}$ specimen grown at $1150^{\circ} \mathrm{C}$ with an acetylene supersaturation of $3 \%$. As shown in Figs. $3 b$ and $3 c$, the sets of facets are of two different types which were referred to as (cubic) C-set and (octahedral) O-set, respectively, in Ref. [6]. Both sets are arranged in a threefold symmetric pattern along the sides of the samples reflecting the $3 \mathrm{~m} 1$ point group symmetry of the $\{111\}$ top faces. The orientations of the two sets, i.e. the directions in which the photographs of Fig. 3 were taken, are indicated in Fig. 1b. The appearance of the curved band shows a high density of macrosteps parallel to the $\langle 110\rangle$ PBCs close to the O-set indicating stabilisation of the surface in only one direction. This differs significantly from the more flattened features close to the C-set, which must be obtained by a stabilisation in two directions for a discrete number of orientations (compare Figs. $3 \mathrm{~b}$ and $3 \mathrm{c}$ ). This indicates that besides the hybrid geometry, $R_{\perp}$ does play a significant role in the stabilization of faces between $\{115\}$ and $\{001\}$.

In general the $\{001\}$ and $\{111\}$ side facets are well developed and give clear reflections in the goniometer telescope. Other faces are still slightly curved resulting in a diffuse reflection or a band of reflections in the optical goniometer. In that case angles with an accuracy not better than within $2^{\circ}$ can be obtained. The curved faces with orientations between $\{113\}$ and $\{117\}$ will be referred to as $\{11 X\} \quad(3<X<7)$. The measurements demonstrated that the faces in the C-sets of the sample shown in Fig. 3 are $\{11 X\},\{001\}$, $\{11 X\}$ and $\{111\}$ from top to bottom and that the O-sets exhibit $\{111\},\{112\}$ and $\{001\}$ faces from 

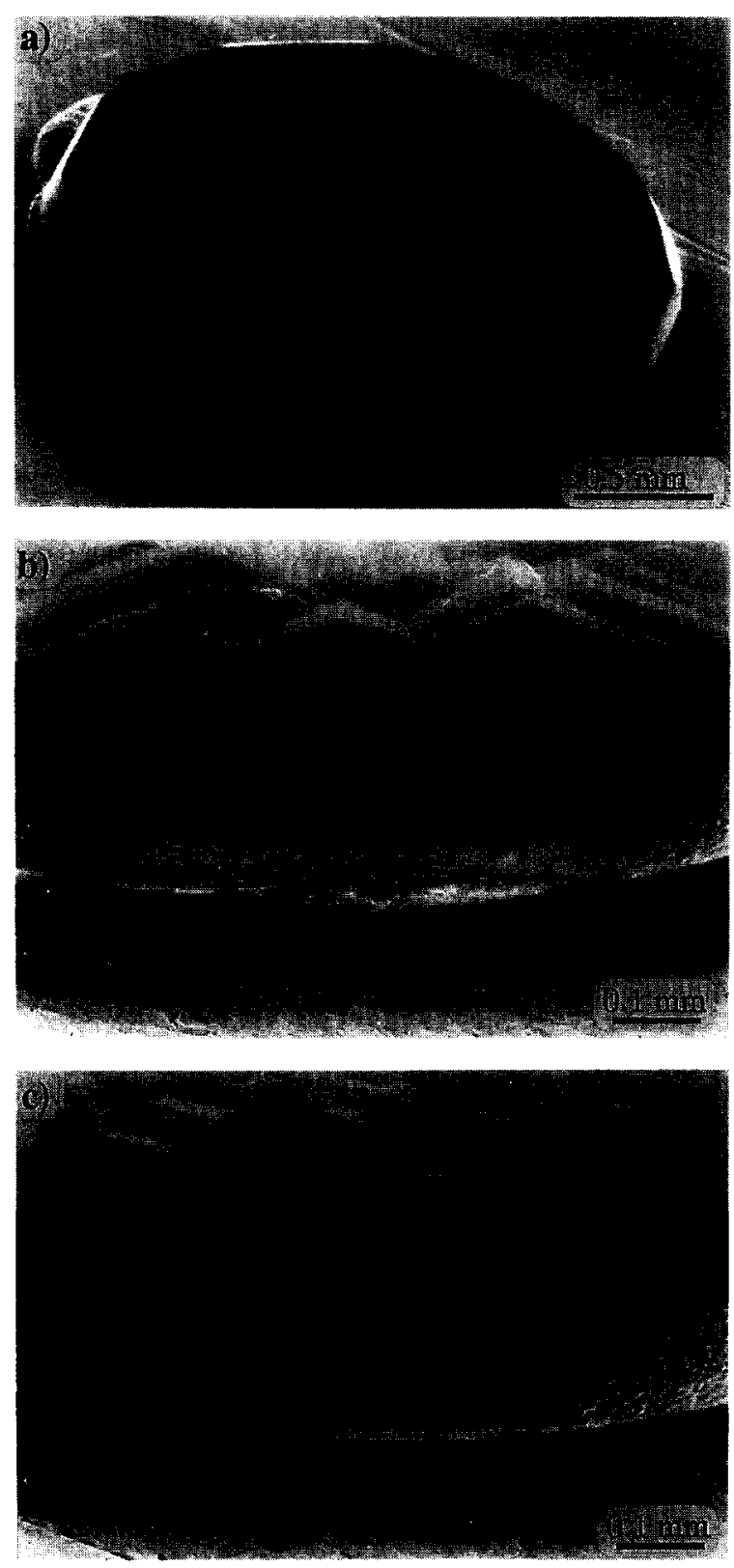

Fig. 3. SEM photographs of a single crystal diamond layer grown on a $\{111\}$ natural diamond substrate $\left(T_{\mathrm{d}}=1150^{\circ} \mathrm{C}\right.$, $S_{\mathrm{ac}}=3 \%$ ). The overview (a) and the detailed pictures of the faceted C- and O-sets, (b) and (c), respectively, were taken at an angle of $\sim 55^{\circ}$ with the $\{111\}$ top face. Note the difference in the curved band close to the two faceted sets and the rough appearance of all other orientations.

top to bottom. Although reported by others [39], in the present case the observed $\{112\}$ faces most probably are just the "translated" sides of the original cylindrical substrate which have not yet grown out of the crystal form. The face has already disappeared from the C-set since the angle between both faces adjacent to the $\{112\}$ orientation in this set $\left(\sim 37^{\circ}\right)$ is smaller than in the O-set $\left(\sim 55^{\circ}\right)$ [6]. In addition, the relatively low degree of stabilization of the $\{112\}$ faces compared to the other side faces is illustrated by the gradual transition to the rough cobbled orientations on both sides of each (see Fig. 3c). The last facets in the sets have angles of more than $90^{\circ}$ with the $\{111\}$ top face and did not directly face the main gas flow. Especially the bottom parts of these facets are ill defined, probably due to transport problems of growth species towards the surface. The large number of flattened areas close to the $\{001\}$ orientations observed in the curved band along the rim of the specimens top face (see Fig. 3b) are not present in the faceted sets. This is explained by the fact that due to the low curvature of the band (radius $\approx 1 \mathrm{~mm}$ ) the flattened areas do not hamper each others development. On the other hand in the region of higher curvature between the $\{111\}$ and $\{001\}$ faces within the faceted sets (radius $\approx 0.2 \mathrm{~mm}$ ) these areas, which correspond with the minima shown in Fig. 2a, have competed with each other and only the most stable, i.e. slowest growing, orientations have survived. The $\{11 X\}$ faces in the $\mathrm{C}$-sets originating from the place with the highest curvature, i.e. the straight corner between the top and sides of the substrate, have the most pronounced appearance.

Fig. 4a gives a schematic cross-section of both sets of facets showing the relative dimensions of the observed faces averaged over the three symmetry equivalent counterparts. The faces, which are elongated along the sides of the substrate, have their minimum size in the plane of the cross-section perpendicular to their $\langle 110\rangle$ PBCs (see Fig. 3). Therefore the dimensions of the facets in this cross-section are a measure for their morphological importance relative to each other. This appears to increase in the order $\{001\}<$ $\{11 X\}<\{111\}$. Fig. $4 b$ shows a cross-section of both sets of facets on a $\{111\}$ sample which was grown at the same supersaturation but at a $100^{\circ} \mathrm{C}$ higher deposition temperature $\left(T_{\mathrm{d}}=1250^{\circ} \mathrm{C}\right)$ than the sample shown in Figs. 3 and $4 \mathrm{a}$. The figure 

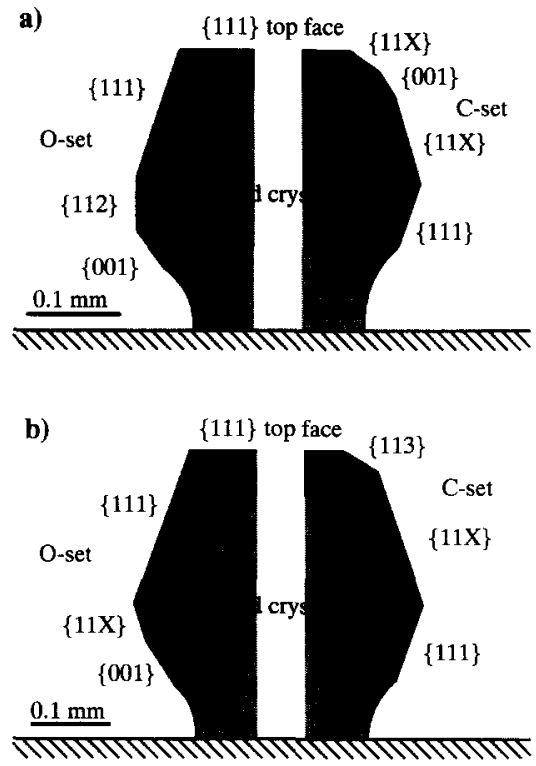

Fig. 4. Schematic cross-sections on scale of two diamond single crystals showing the relative morphological importance of the faces in the different sets of facets. The samples were grown at deposition temperatures of $1150^{\circ} \mathrm{C}$ (a) and $1250^{\circ} \mathrm{C}$ (b), using the same supersaturation $\left(S_{\mathrm{ac}}=3 \%\right)$.

shows that the $\{112\}$ faces in the O-sets, originating from the substrate disappeared by the further development of the $\{11 X\}$ faces. The C-sets differ significantly from those of the $\{111\}$ specimen grown at $1150^{\circ} \mathrm{C}$. The $\{001\}$ faces no longer appear and in addition the difference between the two different $\{11 X\}$ faces in the bands has become more pronounced. Those closest to the top face of the sample now clearly have $\{113\}$ orientations. On the other hand, those further away from the top, where the supersaturation and with it the surface hydrocarbon radical coverage is less, have shifted towards the $\{117\}$ orientations. Close inspection by SEM even reveals a shallow rim indicating that the $\{11 X\}$ faces break up into two separate facets, most probably $\{113\}$ and $\{115\}$ or $\{117\}$ [6]. Compared to the specimen grown at $1150^{\circ} \mathrm{C}$ the morphological importance of the $\{11 X\}$ faces on the sample is increased and is almost as large as that of the $\{111\}$ faces. This is in accordance with the expected increase in stabilization of these faces with increasing tempera- tures, due to a decreased surface coverage with hydrogen and hydrocarbon radicals.

Summarizing, all the observed faces and the curved bands with macrosteps belong to the $\{h h k\}_{h \leq k}$ parts of the $\langle 110\rangle$ zone. Other orientations have a rough appearance (see Figs. $3 b$ and $3 c$ ), including the $\{h h k\}_{h>k}$ orientations where according to the PBC theory stabilization in one direction should occur (see Fig. 1a).

\subsection{Growth on $\{110\}$ substrates}

Along the perimeter of the flame deposited $\{110\}$ samples generally flat $\{111\}$ and $\{001\}$ facets are observed with curved bands of macrosteps along $\langle 110\rangle$ between them [4,5]. These curved bands have sharp rims with the $\{111\}$ side faces, but show a gradual transition towards the $\{001\}$ faces (see Fig. 5a). The reflections of these bands in the $\langle 110\rangle$ zone, as observed by the goniometer, start at angles between $20^{\circ}$ and $25^{\circ}$ with the adjacent $\{111\}$ facet, have a maximum around $30^{\circ}$ corresponding to a $\{113\}$ face and persist all the way towards the $\{001\}$ facet. This behaviour indicates a difference in stability of faces in the $\langle 110\rangle$ zones between $\{111\}$ and $\{113\}$ on one hand and between $\{113\}$ and $\{001\}$ on the other hand. This agrees well with the stabilities expected in case of dimer formation perpendicular to the $\langle 110\rangle$ PBCs. At higher growth temperatures and longer deposition times, the curved bands develop into planar $\{113\}$ facets $[4,7]$. The morphological importance of the $\{113\}$ faces relative to the others is low compared to the $\{111\}$ samples due to a higher acetylene supersaturation in the gas phase (3.9-4.6\% versus $3 \%$ ), which gives a higher surface coverage by hydrocarbon radicals. The $\{110\}$ top faces are smaller than they were before growth. This, and the relatively low morphological importance of the $\{113\}$ faces, indicates that under the applied growth conditions, the final crystal shape will be determined by $\{111\}$ and $\{001\}$ facets, depending on their relative growth rate $[19,21,40]$.

The $\{110\}$ top faces of the samples have a rough appearance due to the formation of microfacets (see Fig. 5b). Rather than a stepped and curved surface due to stabilization in one direc- 
tion by $\langle 110\rangle$ PBCs as expected from a first-order PBC analysis (see Fig. 1a), this orientation is stabilized by microfaceting [5,11]. In this respect $\{110\}$ and more generally the $\{h h k\}_{h>k}$ orienta-
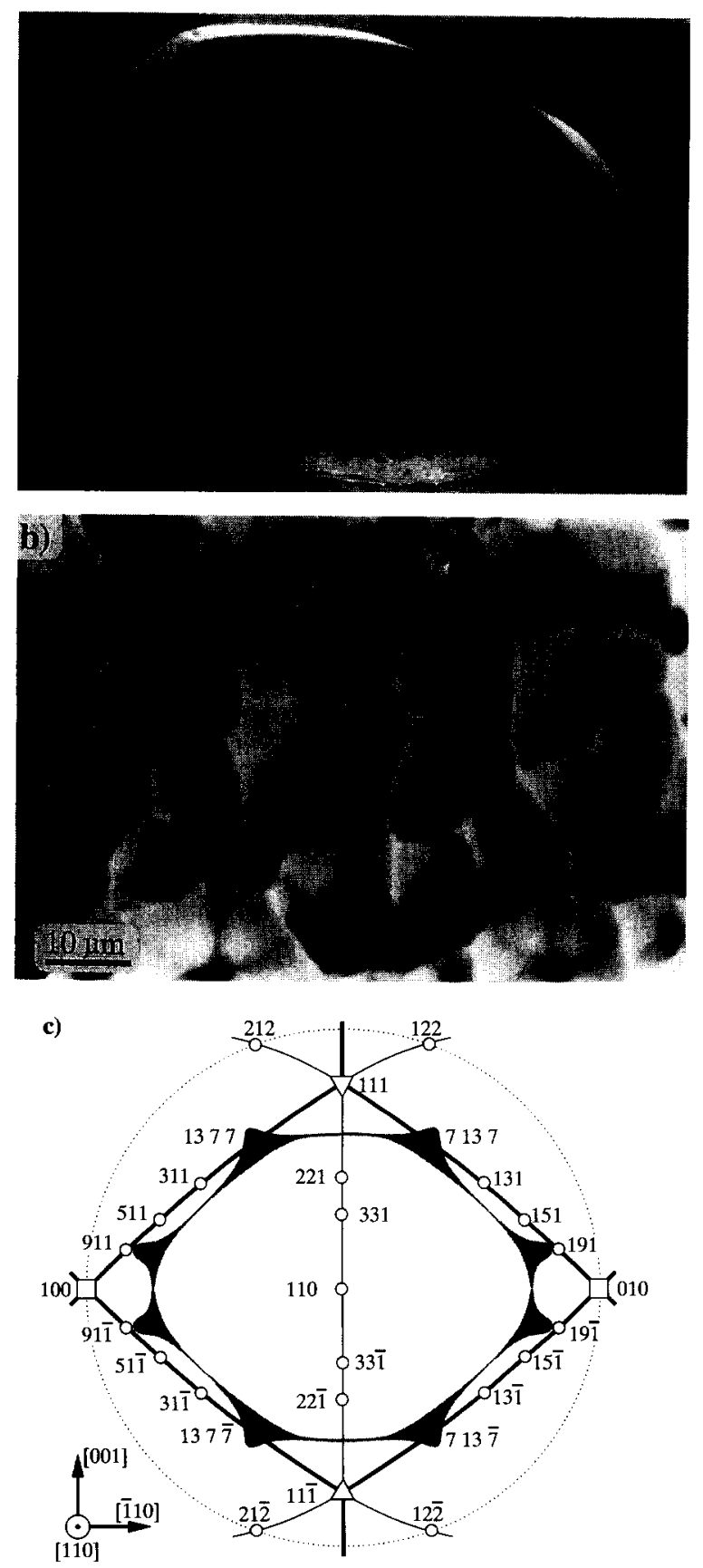

tions behave just as all other orientations with Miller indices other than $\{h h k\}_{h<k}$, as was shown on the $\{111\}$ samples. This demonstrates that the presence of singly directional PBCs in the surface slice under the described growth conditions is not sufficient to give stabilization of any kind if they cannot form a "connected net". The surface free energy of the microfacets must be lower than that of the original $\{110\}$ substrate face, because they develop despite an increase in surface area. The average orientations of the microfacets on such faces were determined by laser reflectometry. From the ovally shaped reflected light pattern, with the most intense reflections from orientations near $\{7713\}$ and $\{119\}$, the distribution of microfacet orientations as indicated by the grey area in the stereographic projection of Fig. 5c was deduced. All microfacet orientations are close to $\{h h k\}_{h<k}$ parts of the $\langle 110\rangle$ zone which is indicated by a bold line. The reflected image certainly does not correspond with $\{h h k\}_{h>k}$ orientations (thin line) as would be expected from a broken bond PBC analysis. The measured orientations show a slight discrepancy with the $\{h h k\}_{h<k}$ parts of the $\langle 110\rangle$ zones, because the microfacets are not fully developed but are somewhat rounded. It seems that the surfaces responsible for the reflections close to $\{119\}$ tend to develop towards $\{001\}$ and those close to $\{7713\}$ towards $\{111\}$ and/or $\{113\}$. The rounded shape of the facets is expected since the reentrant corners between the microfacets in the pits are preferred nucleation sites for steps resulting in the concave shape of the facets.

\footnotetext{
Fig. 5. Morphology of a single crystal diamond layer grown on a $\{110\}$ diamond substrate $\left(T_{\mathrm{d}}=1200^{\circ} \mathrm{C}, S_{\mathrm{ac}}=3.9 \%\right)$. (a) Overview of the sample taken at an angle of about $50^{\circ}$ with the top face (SEM). (b) Perpendicular view on the top face showing the pattern of microfacets (SEM). (c) Distribution of microfacet orientations (grey area) on the top face in a stereographic projection as deduced from LR measurements with the sample aligned along the same orientations as the SEM image shown in (b). All the orientations which can be revealed by LR lay within the dotted circle at $45^{\circ}$ from the central (110) orientation. The $\{h h k\}_{h>k}$ and $\{h h k\}_{h<k}$ parts of the $\langle 110\rangle$ zones are indicated by the thin and bold lines, respectively.
} 


\subsection{Growth on $\{001\}$ substrates}

The sides of the $\{001\}$ specimens, which were grown under similar conditions as the $\{110\}$ samples, are also bounded by $\{111\},\{001\}$ and occasionally by $\{113\}$ facets $[2,3,5,6]$. The top faces of the samples are covered with macrosteps with flat regions in between, giving the samples a high optical transparency. The average distribution of step and terrace surface orientations as deduced from LR measurements, reveal that the macrosteps are generally aligned along the $\langle 110\rangle$ directions (see Fig. 6a). Close examination by high magnification DICM shows that, in conformation with the LR measurements, the steps are usually several micrometers wide and have inclinations varying from $5^{\circ}$ to $20^{\circ}$ with respect to the $\{001\}$ substrate [5]. The terraces between the steps are not exact $\{001\}$ faces but are slightly undulated. These features appear very pronounced on a few specimens which, due to some large defects like inclusions, are locally misoriented from $\{001\}$ (see Fig. 6b). Similar patterns were observed by Everson and Tamor [41] at hillocks generated by what they describe as "gross defects" on diamond layers grown by micro-plasma assisted CVD at $900^{\circ} \mathrm{C}$. As was also argued by these authors [42], only $\mathrm{D}_{\mathrm{A}}$ steps can account for the distinct and abrupt changes in slope of the diamond layers. It seems that the phenomenon is related with a fast generation of steps which is common for flame deposition. Because of the large step fluxes the situation at the crystal surface goes further from equilibrium, were only $D_{B}$ steps are expected [25], so that the reconstruction according to $R_{\perp}$ involving $\mathrm{D}_{\mathrm{A}}$ steps is favoured. These steps pile-up and form one of the faces in the $\langle 110\rangle$ zone between $\{001\}$ and $\{113\}$ which are stabilized in two directions (see Section 2). Due to its stability this face persists and advances slower over $\{001\}$, so that isolated steps or the less stable step faces with orientations closer to the $\{001\}$, which might well correspond to the undulations at the terraces, catch up and join them. Stabilized faces of several types as well as random step patterns are distributed over the top faces of the samples. Therefore the reflected laser light image becomes blurred so the observed discrete behaviour shown
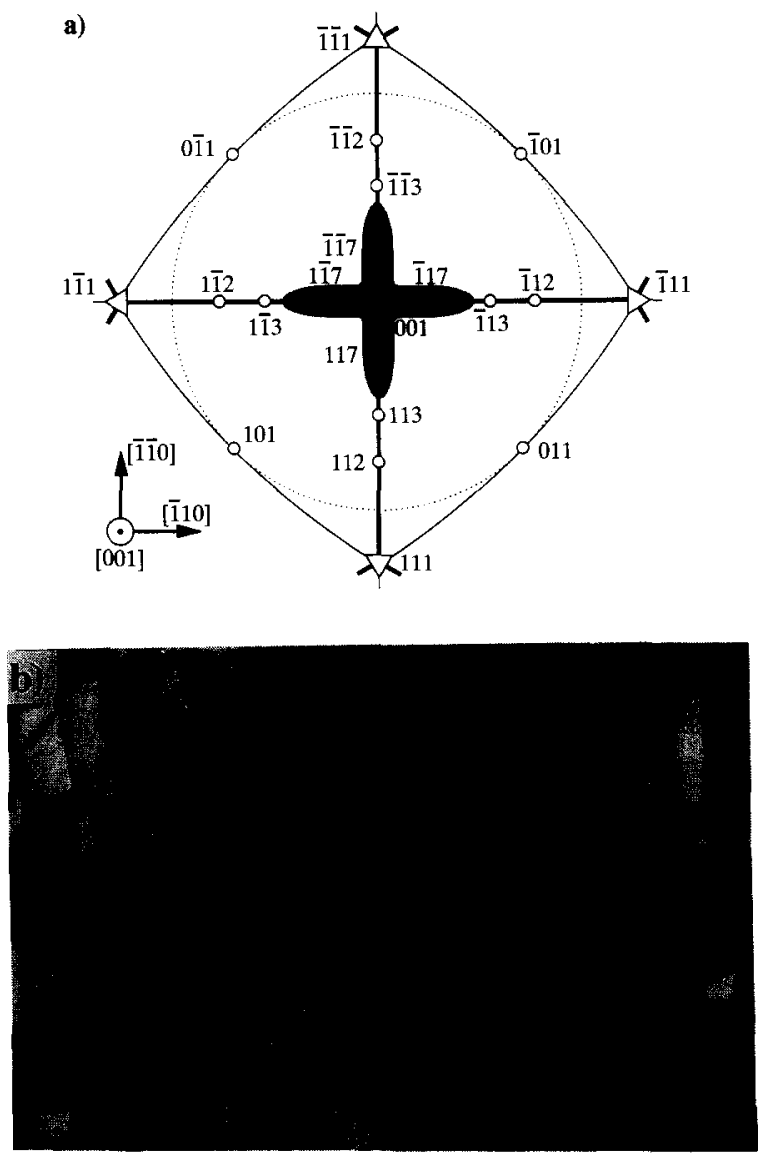

Fig. 6. Morphologies of homoepitaxially grown diamond layers on \{001\} type IIa natural diamond substrates. (a) Typical distribution of macrosteps and terraces (grey area) on the top faces of the $\{001\}$ samples as deduced from LR measurements $\left(T_{\mathrm{d}}=1200^{\circ} \mathrm{C}, S_{\mathrm{ac}}-5.4 \%\right)$. All the orientations which can be revealed by $L R$ lay within the dotted circle at $45^{\circ}$ from the central (001) orientation. The $\{h h k\}_{h>k}$ and $\{h h k\}_{h<k}$ parts of the $\langle 110\rangle$ zones are indicated by the thin and bold lines, respectively. (b) DICM image of the most pronounced appearance of steps and terraces observed on a sample with some inclusions $\left(T_{\mathrm{d}}=1150^{\circ} \mathrm{C}, S_{\mathrm{ac}}=4 \%\right)$. The distribution as obtained by LR measurements and the DICM picture are aligned along the same orientations.

in Fig. $6 \mathrm{~b}$ cannot be recognized in the reflection patterns (see Fig. 6a). Note that the surfaces of the steps aligned along the $\langle 110\rangle$ directions shown in Fig. $6 \mathrm{~b}$ are flat, while these parts of the steps aligned along other directions are rough and break up into small surface areas with orienta- 
tions close to $\{h h k\}_{h \leq k}$. In fact a kind of microfaceting occurs.

\subsection{Nitrogen-vacancy pairs}

The orientation dependent incorporation of impurities was investigated by flame deposition of a thin epitaxial layer on a hemispherical diamond substrate with a $\{110\}$ top orientation [11]. The CL image of the grown layer shown in Fig. 7, reveals blue band A luminescence (dark areas) in the $\{h h k\}_{h \leq k}$ parts of the $\langle 110\rangle$ zones, including the $\{001\}$ and $\{111\}$ faces. Between the $\{111\}$ and $\{001\}$ facets the width of the bands that reveal blue luminescence is largest for orientations close to $\{113\}$. Surprisingly the exact $\{111\}$ orientations give a less intense blue luminescence than the surfaces slightly misoriented from it. Generally, it is assumed that blue band A cathodoluminescence is due to dislocations and is indicative for highly pure diamond. The orange-red $575 \mathrm{~nm}$ system indicative for nitrogen-vacancy pairs predominates the luminescence in all other directions (bright areas).

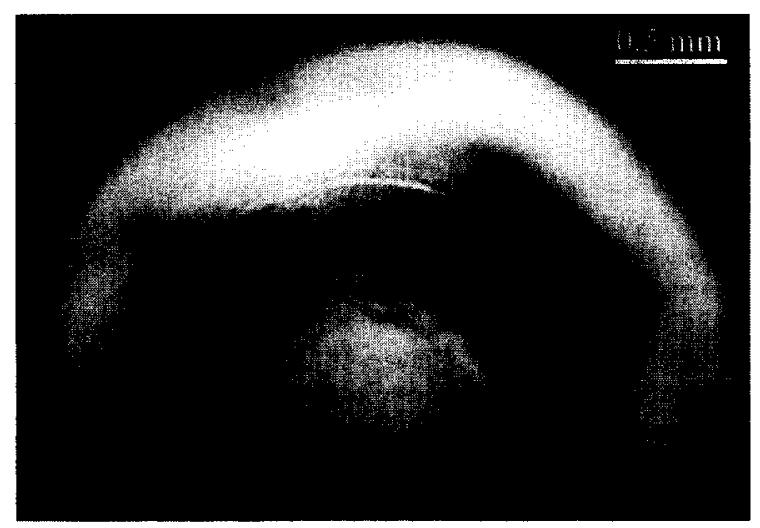

Fig. 7. CL-topograph of a single crystal diamond layer deposited on a hemispheric natural diamond substrate with a $\{110\}$ top orientation $\left(S_{\mathrm{ac}}=3.9 \%, T_{\mathrm{d}}=1150^{\circ} \mathrm{C}\right)$. The bright areas correspond to $575 \mathrm{~nm}$ luminescence from nitrogenvacancy pairs. The darker areas corresponding to blue band $A$ luminescence are restricted to the $\{h h k\}_{h<k}$ parts of the $\langle 110\rangle$ zones between $\{111\}$ and $\{001\}$. From a comparison with the stereographic projection of Fig. $5 \mathrm{c}$ the $\{111\}$ and $\{001\}$ faces can be easily identified by their three- and fourfold symmetric patterns, respectively.
The grown layer on the hemisphere is not thick enough to reveal the same morphological features as described for the cylindrical samples. The CL image however, supports the model described in Section 2, since surfaces with a higher degree of stabilization due to dimer formation are expected to have a higher resistance against incorporation of "foreign" atoms than non-stabilized surfaces. At any rate the luminescent behaviour of crystal volumes grown via the reconstructed surfaces is expected to show similarities. In this respect it is interesting to note that the luminescent characteristics of the exact $\{111\}$ orientations, which are stabilized by interconnected $\langle 110\rangle$ PBCs without the formation of dimers, differs from the $\{h h k\}_{h<k}$ orientations.

In accordance with the sphere-experiment the top faces of the cylindrical $\{110\}$ specimens reveal luminescence of the $575 \mathrm{~nm}$ system, while the $\{111\}$ and $\{001\}$ samples mainly show blue band $A$ luminescence [5]. The blue luminescence of the $\{111\}$ samples originates from slightly misoriented faces, since the $\{111\}$ samples are fully covered by triangular growth hillocks with vicinal faces of the positive form $\{111+\delta\}$ with inclinations of $4^{\circ}[6]$.

\section{Conclusions}

The orientation dependent growth phenomena observed on homoepitaxially flame grown single crystals have been examined by SEM, DICM, optical goniometry, laser reflectometry and CL topography. For all samples the observed faces and the curved bands with macrosteps only occur in the $\{h h k\}_{h \leq k}$ parts of the $\langle 110\rangle$ zone. In this zone the orientations between $\{111\}$ and $\{113\}$ are stabilized in one direction (stepped and curved bands), while a number of discrete orientations between $\{113\}$ and $\{001\}$ are stabilized in two directions (flattened areas). All other orientations have a rough appearance, so no faces or other features occur belonging to the $\{h h k\}_{h>k}$ parts of the $\langle 110\rangle$ zone from which the faces should be $S$-type. From this it can be concluded that the presence of $\langle 110\rangle$ PBCs consisting of nearest neighbour covalent bonds in only one direction is not sufficient to stabilize the surface. Some kind 
of interwoven net has to be formed to obtain this stabilization.

It is proposed that for the orientations with Miller indices $\{h h k\}_{h<k}$, in addition to the $\langle 110\rangle$ PBCs, dimer formation perpendicular to these PBCs is responsible for such a net. The fact that the observed faces between $\{113\}$ and $\{001\}$ are still somewhat curved perpendicular to the $\langle 110\rangle$ PBCs, indicates that in addition also the other reconstruction geometries considered in this study occur. On the basis of this assumption a model has been developed which explains the observed growth phenomena very well. Therefore it can be concluded that surface reconstruction by dimer formation plays a key role in the morphology of CVD grown diamond. Even the $\{111\}$ orientations with connected PBCs in three different directions in a surface slice appear to be stabilized by dimer formation which leads to the development of vicinal faces $\{111+\delta\}$.

\section{Acknowledgments}

The authors wish to thank Mr. W. Elst and Mr. H. Geurts for their assistance with the scanning electron microscope. Furthermore Dr. G. Janssen is acknowledged for useful discussions. This work was financially supported by the European Community Brite Euram project BE 5099-92 under contract BRE-2-0147.

\section{References}

[1] Y. Hirose and N. Kondo, Extended Abstracts, 35th Jap. Appl. Phys. Spring Meeting, 1988, p. 434.

[2] K.A. Snail and L.M. Hanssen, J. Crystal Growth 112 (1991) 651.

[3] K.A. Snail, C.L. Vold, C.M. Marks and J.A. Freitas Jr., Diamond and Related Mater. 1 (1992) 180.

[4] G. Janssen, J.J. Schermer, W.J.P. van Enckevort and L.J. Giling, J. Crystal Growth 125 (1992) 42.

[5] J.J. Schermer, W.J.P. van Enckevort and L.J. Giling, Diamond and Related Mater. 3 (1994) 408.

[6] J.J. Schermer, P. Alers and L.J. Giling, J. Appl. Phys. submitted.

[7] K.A. Snail, Z.P. Lu, R. Weimer, J. Heberlein, E. Pfender and L.M. Hanssen, J. Crystal Growth 137 (1994) 676.

[8] P. Hartman, Z. Krist. 121 (1965) 78.
[9] J.G.E. Gardeniers, W.E.J.R. Maas, R.Z.C. van Meerten and L.J. Giling, J. Crystal Growth 96 (1989) 821.

[10] J.G.E. Gardeniers, W.E.J.R. Maas, R.Z.C. van Meerten and L.J. Giling, J. Crystal Growth 96 (1989) 832.

[11] W.J.P. van Enckevort, G. Janssen, W. Vollenberg, J.J. Schermer and L.J. Giling, Diamond and Related Mater. 2 (1993) 997.

[12] P. Bennema and J.P. van der Eerden, in: Morphology of Crystals, Ed. I. Sunagawa (Terrepub, Tokyo, 1987) p. 1.

[13] P. Hartman and W.G. Perdok, Acta Cryst. 8 (1955) 49, 521,525 .

[14] J.K. Mackenzie, A.J.W. Moore and J.F. Nicholas, J. Phys. Chem. Solids 23 (1962) 185.

[15] D.J. Chadi, Phys. Rev. B 29 (1984) 785.

[16] C. Rottmann and M. Wortis, Phys. Rep. 103 (1984) 59.

[17] C. Herring, Phys. Rev. 82 (1951) 87.

[18] G.A. Wolff, Z. Physik. Chem. Neue Folge 31 (1962) 1.

[19] B.V. Spitsyn, L.L. Bouilov and B.V. Derjaguin, J. Crystal Growth 52 (1981) 219.

[20] W.J.P. van Enckevort, G. Janssen and L.J. Giling, J. Crystal Growth 113 (1991) 295.

[21] R.E. Clausing, L. Heatherly, L.L. Horton, E.D. Specht, G.M. Begun and Z.L. Wang, Diamond and Related Mater. 1 (1992) 411.

[22] L.J. Giling and W.J.P. van Enckevort, Surface Sci. 161 (1985) 567.

[23] T. Tsuno, T. Imai, Y. Nishibayashi, K. Hamada and N. Fujimori, Jap. J. Appl. Phys. 30 (1991) 1063.

[24] L.F. Sutcu, M.S. Thompson, C.J. Chu, R.H. Hauge, J.L. Margrave and M.P. D'Evelyn, Appl. Phys. Lett. 60 (1992) 1685.

[25] D.J. Chadi, Phys. Rev. Lett. 59 (1987) 1691.

[26] T. Tsuno, T. Tomikawa, S. Shikata, T. Imai and N. Fujimori, Appl. Phys. Lett. 64 (1994) 572.

[27] C. Herring, in: Structure and Properties of Solid Surfaces, Eds. R. Gomer and C.S. Smith (University of Chicago Press, Chicago, 1952) p. 5.

[28] G.D. Kubiak, A.V. Hamza, R.H. Stulen, E.C. Sowa, K.W. Kolasinski and M.A. Van Hove, in: New Diamond Science and Technology, Eds. R. Messier, J.T. Glass, J.E. Butler and R. Roy (Materials Research Society, Pittsburgh, PA, 1991) p. 21.

[29] G. Janssen, Homoepitaxial Diamond synthesized by CVD processes, Thesis, University of Nijmegen, 1994.

[30] J.J. Schermer, J.E.M. Hogenkamp, G.C.J. Otter, G. Janssen, W.J.P. van Enckevort and L.J. Giling, Diamond and Related Mater. 2 (1993) 1149.

[31] S.J. Harris, Appl. Phys. Lett. 56 (1990) 2298.

[32] L.R. Martin and M.W. Hill, J. Mater. Sci. Lett. 9 (1990) 621.

[33] M. Frenklach and K.E. Spear, J. Mater. Res. 3 (1988) 133.

[34] M.H. Loh and M.A. Cappelli, in: Proc. 3rd Int. Symp. on Diamond and Related Mater., Eds. J.P. Dismuskus, K.V. Ravi, B. Lux and N. Setaka (Electrochemical Society, Pennington, NJ, 1993) p. 17.

[35] M.A. Cappelli and M.H. Loh, Diamond and Related Mater. 3 (1994) 417. 
[36] N.A. Pangarov, in: Rost Kristallov, Vol. 10, Ed. N.N. Sheftal (Nauka, Moscow, 1974).

[37] J.J. Schermer, W.A.L.M. Elst and L.J. Giling, Diamond and Related Mater., submitted.

[38] D.J. Marshall, Cathodoluminescence of Geological Materials (Unwin Hyman, London, 1988).

[39] A. Badzian and T. Badzian, Diamond and Related Mater. 2 (1993) 147.
[40] C. Wild, R. Kohl, N. Herres, W. Müller-Sebert and P. Koidl, Diamond and Related Mater. 3 (1994) 373.

[41] M.P. Everson and M.A. Tamor, J. Mater. Res. 7 (1992) 1438.

[42] M.A. Tamor and M.P. Everson, J. Mater. Res. 8 (1993) 1770 . 